\title{
Efficacy of Neem, Tithonia and Tephrosia Leaf Extracts in Management of Root-Knot Nematodes in French Beans (Phaseolus vulgaris L.)
}

\author{
Joshua K. Njenga \\ Chuka University, P.O. Box 109-60400, Chuka, Kenya \\ Geofrey K. Gathungu (Corresponding author) \\ Chuka University, P.O. Box 109-60400, Chuka, Kenya \\ E-mail: gkgathungu@yahoo.com; joshuakinene72@gmail.com \\ Jesca N. Mbaka \\ Kenya Agricultural and Livestock Research Organization, Kandara P.O. Box 220-01000, \\ Thika, Kenya
}

Received: July 26, 2019

Accepted: August 12, 2019

Published: August 21, 2019

doi:10.5296/jas.v7i3.15313

URL: https://doi.org/10.5296/jas.v7i3.15313

\begin{abstract}
Root-knot nematodes (Meloidogyne spp.) are a major problem in French bean production within the smallholder farming systems. Control of root-knot using synthetic nematicides is not viable due to environmental concerns relating to their toxic residues. There is need to develop alternative control options that will promote soil health and reduce parasitic nematode densities. A study was conducted to determine the efficacy of Neem (Azadirachta indica A. Juss), Tithonia (Tithonia diversifolia) and Tephrosia (Tephrosia purpurea) leaf extracts in management of root-knot. Controlled lath house and field trials were conducted where the treatments were extracts from Neem, Tithonia and Tephrosia at different concentration levels of $25 \mathrm{ml} / \mathrm{L}, 50 \mathrm{ml} / \mathrm{L}$ and $100 \mathrm{ml} / \mathrm{L}$ ). Vydate (Oxamyl 10\%) a synthetic nematicide served as a standard positive control while treatments with no extracts application and no nematode application served as negative controls. The treatments in the lath house were arranged in completely randomized design while the field trials were arranged in randomized complete block design. Fench beans were planted on nematode infested soils and data on root galling indices and yield components was collected. Data collected was subjected to analysis of variance and significantly different means separated using Tukey's
\end{abstract}


Studentized Range Test at $P=0.05$. The extracts evaluated reduced root galling with their efficacy being similar to that of Vydate ${ }^{\circledR}$ (Oxamyl 10\%) which was used as a positive control. Neem extracts treatments had the lowest mean galling index of the extracts, followed by Tithonia. Root-knot nematode galling indices were highest in the untreated control at 10 both in trial I and trial II. Treatment trials from Neem and Tithonia at concentration levels of 100 $\mathrm{ml} / \mathrm{L}$ resulted in highest yield of French beans while yield from the untreated plants were the lowest. The results indicate extracts can be adopted to suppress root-knot nematodes.

Keywords: neem, tithonia, tephrosia, phytochemicals, French beans, nematodes, phytotoxicity

\section{Introduction}

Globally, yield losses due to arthropods, diseases, and weeds are estimated to account for about 35\% in major crops (Dougoud et al., 2019). Root-knot nematodes (RKNs) are a malignant soilborne curse to vegetables, which undermine their production and few farmers are aware of them or the damage they cause (Coyne et al., 2018). They are a major constraint to successful vegetable production all over the world, causing severe damage that leads to dramatic yield losses (Sujatha et al., 2017). Existing control measures involving applications of chemical nematicides are not viable in the medium to long term due to environmental concerns relating to toxic residues (Muhammad et al., 2017). There is therefore a need to develop alternative control options for integrated parasitic nematode management that will promote soil health and reduce parasitic nematode densities (Emmanuel et al., 2017). Meloidogyne spp. is a major problem in French beans productions in many French bean production systems in Kenya.

Root-knot nematodes are small unsegmented worms and are mostly microscopic and they cause significant damage in almost all crops (Perry et al., 2009). The Meloidogyne spp. attacks over 140 species of more than 115 plant genera in 46 families (Patil et al., 2017). The most characteristic symptom of root knot nematodes infection is the presence of giant cells on the roots known as galls or knots while the aboveground symptoms include the yellowing of leaves, defoliation, stunting and wilting (Jang et al., 2019). Nematicides are recommended for the management of nematodes but they are highly toxic compounds that have very low LD 50 values (Odeyemi et al., 2013). In many parts of the world most of the nematicides are banned because of their harmful effect on humans and environment. Chemical nematicides are one of the fastest and effective nematode control methods (Wachira et al., 2009) but there is need to develop alternative methods of control that are cheap, environmentally friendly and not harmful to humans (Priya and Pandiyan, 2019).

Therefore, it has become an important issue to find alternative control strategies that can lead to effective nematode control and also assure the safety of the consumers of horticultural products. Many plants are known to have nematicidal properties which may be utilized as organic amendments or bio-pesticides. Use of plant extracts on eggs and larvae to reduce the nematode population is promising in the crop protection against root-knot nematodes. Plant extracts having the nematicidal properties may also enhance the plant growth. Patil et al. (2017) tested the extracts of Neem for their effects on larval mortality of nematode and the 
extracts exhibited significant mortality. Oyinlola (2017) reported that aqueous extracts from Tithonia and Tephrosia reduced nematode populations in the soil with corresponding increases in plant height, plant leaf and fruit yield. This study aimed at evaluating the efficacy of Neem, Tithonia and Tephrosia extracts which are readily available in management of root-knot nematodes in French beans.

\section{Material and Methods}

Study site

The experiments were conducted at Chuka University. The area is situated approximately $186 \mathrm{~km}$ from Nairobi along the Nairobi-Meru highway and lies at an approximately $0^{\circ} 19.9896^{\prime}$ South latitude, 37³8.7522' East longitude and $1452 \mathrm{~m}$ elevation above the sea level. The climate is warm temperate and experiences a bimodal rainfall of about 1000-1599 $\mathrm{mm}$. The average annual temperature is $19.5^{\circ} \mathrm{C}$. The long rainy season occurs in March to July and the short rains occur from October to December. The soils at the University farm are nitisol.

\section{Acquisition of Test Plant Material}

Fresh shoot leaves of Tithonia and Tephrosia were collected from open fields around Chuka University. Commercial Neem leaf formulation (Nimbecidine) for comparison with extracted Neem was purchased from a local Agro-chemical shop. Neem leaves were sourced from the coastal region where Neem trees grow extensively. The plant species were taxonomically identified and authenticated by using the available colour pictures followed by description and identification characters. The plants were identified and authenticated by a taxonomist and voucher specimen of the plant deposited at Chuka University Chemistry laboratory with voucher No. MSC/HORT5/11/2017.

\section{Extraction and Storage of Crude Extracts}

Extracts were prepared as described by Oloruntola et al. (2017). The fresh leaves were air-dried in shade for 2 weeks then coarsely powdered with a mechanical grinder separately. Two hundred grams of dried powder of each plant species were weighed and dissolved in 500 $\mathrm{ml}$ of $95 \%$ ethanol in an Erlenmeyer flask for elucidation. After 24 hours of soaking the solutions were filtered through two layers of cheese-cloth gauze and Whitman's No. 2 filter paper before the filtrates were subjected to evaporation using a rotary flash evaporator under reduced pressure at $60^{\circ} \mathrm{C}$ for 60 minutes to concentrate the extract and remove the ethanol. Extracts were stored in airtight container in refrigerator at $10^{\circ} \mathrm{C}$. Concentrations were prepared following Muhammad et al. (2017) procedure. Concentration of 2.5\% (25 ml/L) 5\% $(50 \mathrm{ml} / \mathrm{L})$ and $10 \%(100 \mathrm{ml} / \mathrm{L})$ were prepared separately by adding $2.5 \mathrm{ml}, 5 \mathrm{ml}$ and $10 \mathrm{ml}$ of the extract residuals with $5 \mathrm{ml}$ of acetone to enhance dissolution and made up to $100 \mathrm{ml}$ by adding tap water.

\section{Preparation of Root-knot Nematode Inoculum}

For extraction of juveniles the roots were gently washed to remove adhering soil particles. The washed roots were cut into small bits of $2.5 \mathrm{~cm}$ longitudinally then placed over tissue 
paper spread on a wire gauge and kept in a Petri plate filled with water. The level of water was maintained in Petri plate and left undisturbed for $48 \mathrm{hrs}$. Later, the suspension in the Petri plate was collected and observed for nematodes using a light-binocular microscope.

\section{Experimental Design and treatment application}

The treatments were Tithonia, Neem and Tephrosia leaf extracts each at a concentration of $2.5 \%$ (25 ml/L), 5\% (50 ml/L ) and 10\% (100 ml/L), Vydate (Oxamyl 10\%) and Commercial Neem leaf formulation (Nimbecidine) served as a standard positive control. Distilled water was used as a standard negative control. In all these treatments $50 \mathrm{ml}$ of the extracts was used as an inoculant. Lath house experiment was laid out in completely randomized design with 3 replications while the field trials were laid out in randomized complete block design with three replications

The first trial was conducted from September 2017 to January 2018. The experiment was repeated as trial two from February to August 2018. There were a total of 225 pots in the lath house experiment. Each of the 15 treatments had 5 potted plants which were replicated randomly three times. The field plots measured 1.5 by $1 \mathrm{~m}$ and a spacing of $30 \times 15 \mathrm{~cm}$ was used making a total of 40 plants. In both lath-house and field experiment inoculation with the nematodes was done before planting. Inoculation was done by pipetting 100 root-knot nematodes masses suspension using a graduated pipette and covered lightly. One week after inoculation the extracts were applied at $50 \mathrm{ml}$ per pot/plant using a syringe. A final application was done four weeks after the first application.

\section{Results}

\section{Number of Pods in Lath house Potted Plants}

It was observed that French beans number of pods among different extract treatments differed significantly $(P=0.05)$ at 55,63, 69 and 76 days after emergence (DAE). The results of this study showed that crude extracts treated French beans plants produced significantly higher French bean number of pods compared to untreated controls infested with root-knot nematodes. From the lath house study the untreated control attained the least number of pods at 9.5 and 9.7 pods in trial I and trial II, respectively (Table 1).

\section{Number of Pods in a Field Experiment}

In the field the number of pods under selected extracts treatments to Meloidogyne spp. differed significantly $(P=0.05)$ among the different treatments. The extract produced significantly high French bean number of pods, which was statistically significant compared to untreated control. Untreated controls recorded the least mean number of pods at 9.4 and 9.5 in trial I and trial II, respectively. Neem at $100 \mathrm{ml} / \mathrm{L}$ attained the highest average mean number of pods at 17.4 and 17.6 in trial I and trial II, respectively. Oxymyl (positive control) attained 15.3 and 15.5 pods in trial I and trial II, respectively (Table 2). 
Table 1. Effects of Different Extracts on Lath house Number of Pods

\begin{tabular}{|c|c|c|c|c|c|c|c|c|c|c|c|c|c|c|c|c|}
\hline \multicolumn{17}{|c|}{ Extracts Concentration Rate } \\
\hline DAE & $\mathrm{N} 25$ & $\mathrm{~N} 50$ & N10 & $\mathrm{Nb} 25$ & $\mathrm{Nb50}$ & $\mathrm{Nb} 10$ & $\mathrm{~T} 25$ & T50 & T10 & $\mathrm{Te} 25$ & $\mathrm{Te} 50$ & Te10 & Oxy & $\mathrm{CN}$ & Cwit & MSD \\
\hline \multicolumn{17}{|c|}{ Trial I } \\
\hline 55 & $22 \mathrm{ac}^{*}$ & $24.6 \mathrm{a}$ & $23.6 a b$ & $24.6 \mathrm{a}$ & $23.3 \mathrm{ac}$ & $21.1 \mathrm{ec}$ & $22.6 \mathrm{ac}$ & $19.7 \mathrm{de}$ & $20.4 \mathrm{ce}$ & $21.0 \mathrm{ec}$ & $18.4 \mathrm{e}$ & $22.2 \mathrm{acd}$ & $22.1 \mathrm{ec}$ & $24.2 \mathrm{a}$ & $15.1 \mathrm{f}$ & 21.25 \\
\hline 62 & $21.7 \mathrm{a}$ & $22.3 \mathrm{a}$ & $20.4 \mathrm{a}$ & $21.3 \mathrm{a}$ & $20.7 \mathrm{a}$ & $22.1 \mathrm{a}$ & $20.1 \mathrm{a}$ & $24.2 \mathrm{a}$ & $22.6 a$ & $22.8 \mathrm{a}$ & $20.6 \mathrm{a}$ & $20.86 a$ & $20.2 \mathrm{a}$ & $21.1 \mathrm{a}$ & $16.3 b$ & 4.81 \\
\hline 69 & $16.0 \mathrm{ab}$ & $17.0 \mathrm{a}$ & $10.4 \mathrm{ac}$ & $14.2 \mathrm{ab}$ & $15.6 \mathrm{ab}$ & $14.2 \mathrm{ab}$ & $16.2 \mathrm{ab}$ & $16.8 \mathrm{a}$ & $16.4 \mathrm{ab}$ & $15.8 \mathrm{ab}$ & $15.8 \mathrm{ab}$ & $7.93 b c$ & $13.2 \mathrm{abc}$ & $10.4 \mathrm{ab}$ & $4.93 \mathrm{c}$ & 8.61 \\
\hline 76 & $11.2 \mathrm{ab}$ & $13.7 \mathrm{ab}$ & $18.3 \mathrm{ab}$ & $11.46 a b$ & $17.2 \mathrm{ab}$ & $15.6 \mathrm{ab}$ & $16.7 \mathrm{ab}$ & $15.7 \mathrm{ab}$ & $19.1 \mathrm{a}$ & $17.3 \mathrm{ab}$ & $18.2 \mathrm{ab}$ & $17.3 \mathrm{ab}$ & $17.9 \mathrm{ab}$ & $15.6 \mathrm{ab}$ & $10.2 b$ & 5.95 \\
\hline 83 & $8.6 \mathrm{a}$ & $8.0 \mathrm{a}$ & $8.8 \mathrm{a}$ & $7.9 \mathrm{a}$ & $7.2 \mathrm{a}$ & $7.2 \mathrm{ab}$ & $4.8 \mathrm{ab}$ & $4.2 \mathrm{ab}$ & $5.8 \mathrm{ab}$ & $9.8 \mathrm{a}$ & $7.8 \mathrm{a}$ & $7.3 \mathrm{a}$ & $7.1 \mathrm{ab}$ & $6.2 \mathrm{ab}$ & $1.4 \mathrm{~b}$ & 5.9 \\
\hline \multicolumn{17}{|c|}{ Trial II } \\
\hline 55 & $20.1 \mathrm{ac}$ & $25.1 \mathrm{a}$ & $24.0 \mathrm{ab}$ & $25.2 \mathrm{a}$ & $24.2 \mathrm{ac}$ & $22.1 \mathrm{ec}$ & $23.2 \mathrm{ac}$ & 20.1de & $21.1 \mathrm{e}$ & 22.1ce & $19.1 \mathrm{ad}$ & 23.1ac & $23.1 \mathrm{ec}$ & $25.1 \mathrm{a}$ & $14.2 \mathrm{f}$ & 20.11 \\
\hline 62 & $21.2 \mathrm{a}$ & $23.1 \mathrm{a}$ & $21.2 \mathrm{a}$ & $21.3 \mathrm{a}$ & $20.7 \mathrm{a}$ & $22.1 \mathrm{a}$ & $21.2 \mathrm{a}$ & $22.1 \mathrm{a}$ & $23.1 \mathrm{a}$ & $22.8 \mathrm{a}$ & $21.1 \mathrm{a}$ & $21.1 \mathrm{a}$ & $20.1 \mathrm{a}$ & $22.2 \mathrm{a}$ & $16.2 b$ & 5.11 \\
\hline 69 & $17.0 \mathrm{ab}$ & $17.1 \mathrm{a}$ & $17.1 \mathrm{ac}$ & $14.2 \mathrm{ab}$ & $15.2 \mathrm{ab}$ & $15.2 \mathrm{ab}$ & $17.2 \mathrm{ab}$ & $13.2 \mathrm{ab}$ & $10.4 \mathrm{ab}$ & $8.55 \mathrm{ab}$ & $13.2 \mathrm{ab}$ & $9.32 b c$ & $14.23 \mathrm{ab}$ & $11.2 \mathrm{ab}$ & $5.12 \mathrm{c}$ & 9.33 \\
\hline 76 & $13.2 \mathrm{ab}$ & $11.5 \mathrm{ab}$ & $19.2 \mathrm{ab}$ & $12,1 \mathrm{ab}$ & $18.2 \mathrm{ab}$ & $16.2 \mathrm{ab}$ & $19.1 \mathrm{a}$ & $15.7 \mathrm{ab}$ & $21.1 \mathrm{a}$ & $18.3 \mathrm{ab}$ & $19.2 \mathrm{ab}$ & $18.3 \mathrm{ab}$ & $18.1 \mathrm{ab}$ & $15.3 b$ & $11.2 \mathrm{~b}$ & 5.54 \\
\hline 83 & $7.5 \mathrm{a}$ & $7.5 \mathrm{a}$ & $7.4 \mathrm{a}$ & $7.9 \mathrm{a}$ & $6.9 \mathrm{a}$ & $7.6 \mathrm{ab}$ & $5.2 \mathrm{ab}$ & $4.4 \mathrm{ab}$ & $5.9 \mathrm{ab}$ & $8.4 \mathrm{a}$ & $8.5 \mathrm{a}$ & $6.9 \mathrm{a}$ & $6.8 \mathrm{ab}$ & $5.8 \mathrm{ab}$ & $2.1 \mathrm{~b}$ & 4.7 \\
\hline
\end{tabular}

*Means with the same letter(s) along the row for DAP and the column for Extracts are not significantly different at $\mathrm{P} \leq 0.05$ by Tukey's test. KEY: $\mathrm{N}=$ Neem, $\mathrm{T}=$ Tithonia, $\mathrm{Te}=$ Tephrosia, Oxy =Oxamyl, $\mathrm{CNO}=\mathrm{Control}, \mathrm{C}$ with $=$ Control with nematodes, the numbers 25,50 , and 100 represent concentration of extract in $\mathrm{ml} / \mathrm{L}$

\section{Fresh Pod Weight in a Lath house Potted Plants}

From the study it was observed that French beans pods weight differed significantly among the different extracts treatments at 69, 76 and 83 DAP under lath house experiment. The results of this study showed that French beans plants treated with the extract produced significantly higher French bean pods weight when compared to the untreated root-knot nematode infested plants. From the study it was observed that the untreated control treatment attained the least pods weight of $24.9 \mathrm{~g}$ and $28.0 \mathrm{~g}$ in trial I and trial II respectively. Neem at $50 \mathrm{ml} / \mathrm{L}$ attained the highest average mean pods weight of $50.9 \mathrm{~g}$ in both trial I and trial II. Oxymyl (positive control) attained an average weight of $48.7 \mathrm{~g}$ and $49.3 \mathrm{~g}$ in trial I and trial II, respectively. Neem, Tithonia and Oxymyl compared in suppression of which resulted in increase in French bean pod weight (Table 3). This can be attributed to better control of the root knot nematodes especially in early stages, therefore, allowing the French bean crop to grow with vigor resulting in French bean pod weight increase. In both trials, Oxamyl gave lower French bean pod weight. Although Tephrosia would be expected to boost French bean pod weight, it seems their effect was overwhelmed by the significantly high populations. Generally French beans pod weight from plots treated with the extracts were higher than the untreated control showing the potential of increasing pod weight.

\section{Pods Weight in a Field Experiment}

In the field experiment French beans pods weight under extracts treatments differed significantly $(P=0.05)$. The study showed that the untreated control treatment severely infested by the root-knot nematodes attained the least pods weight of $28.3 \mathrm{~g}$ and $32.6 \mathrm{~g}$ in trial I and trial II, respectively. Neembecidine at $100 \mathrm{ml} / \mathrm{L}$ treatments attained the highest pod weight of $60.5 \mathrm{~g}$ in trial I while Tephrosia treatments at $100 \mathrm{ml} / \mathrm{L}$ attained the highest pods weight of $62.2 \mathrm{~g}$. Oxymyl (positive control) attained pod weight of $58.2 \mathrm{~g}$ and $59.0 \mathrm{~g}$ in trial I and trial II, respectively (Table 4). In both trials, Oxamyl gave lower French bean pod weight. 
Although Tephrosia would be expected to boost French bean pod weight, it seems their effect was overwhelmed by the significantly high root-knot nematodes populations. Generally French beans pod weight from plots treated with the extracts were higher than the untreated control showing the potential of increasing pod weight.

Table 2. Effects of Different Extracts on Field Number of Pod

\begin{tabular}{|c|c|c|c|c|c|c|c|c|c|c|c|c|c|c|c|c|}
\hline \multicolumn{17}{|c|}{ Extracts Concentration Rates } \\
\hline DAE & $\mathrm{N} 25$ & N50 & N100 & $\mathrm{Nb} 25$ & $\mathrm{Nb50}$ & $\mathrm{Nb} 100$ & $\mathrm{~T} 25$ & T50 & $\mathrm{T} 100$ & $\mathrm{Te} 25$ & $\mathrm{Te} 50$ & $\mathrm{Te} 100$ & Oxy & $\mathrm{CNO}$ & CWith & MSD \\
\hline \multicolumn{17}{|c|}{ Trial I } \\
\hline 55 & 22.ce* & $20.3 \mathrm{ce}$ & 21.6de & $22.6 \mathrm{ac}$ & $21.7 \mathrm{ec}$ & $23.5 \mathrm{ac}$ & $24.3 \mathrm{a}$ & $24.5 \mathrm{a}$ & $24.1 \mathrm{ab}$ & $24.5 \mathrm{a}$ & $24.1 \mathrm{ab}$ & $22.3 \mathrm{ec}$ & $19.9 \mathrm{f}$ & 21.6ed & $14.1 \mathrm{~g}$ & 1.87 \\
\hline 62 & $20.7 \mathrm{ab}$ & 21.0ab & $20.3 \mathrm{ab}$ & $21.4 \mathrm{a}$ & $17.4 \mathrm{~b}$ & $21.6 \mathrm{a}$ & $20.1 \mathrm{ab}$ & $21.6 \mathrm{a}$ & $21.7 \mathrm{a}$ & $22.3 \mathrm{~cd}$ & $23.0 \mathrm{ac}$ & $21.6 \mathrm{ce}$ & $19.9 \mathrm{f}$ & $21.6 \mathrm{ec}$ & $14.1 \mathrm{~g}$ & 1.8 \\
\hline 69 & $13.6 \mathrm{ab}$ & $14.1 \mathrm{ab}$ & $13.4 \mathrm{ab}$ & $14.7 \mathrm{ab}$ & $13.6 \mathrm{ab}$ & $11.06 \mathrm{bc}$ & $13.3 \mathrm{ab}$ & $15.3 \mathrm{ab}$ & $12.8 \mathrm{ac}$ & $16.8 \mathrm{a}$ & $17.2 \mathrm{a}$ & $17.4 \mathrm{a}$ & $15.6 \mathrm{ab}$ & $13.5 \mathrm{ab}$ & $7.2 \mathrm{c}$ & 5.7 \\
\hline 76 & $11.5 \mathrm{~cd}$ & $17.6 \mathrm{ab}$ & $13.3 b c$ & $17.0 \mathrm{ac}$ & $15.6 \mathrm{ac}$ & $18.3 \mathrm{ab}$ & $17.7 \mathrm{ab}$ & $16.7 \mathrm{ac}$ & $16.7 \mathrm{ac}$ & $19.4 \mathrm{a}$ & $13.3 \mathrm{bc}$ & $18.7 \mathrm{ab}$ & $15.8 \mathrm{ac}$ & $15.16 \mathrm{ac}$ & $6.7 \mathrm{~d}$ & 4.7 \\
\hline 83 & $7.5 \mathrm{a}$ & $8.1 \mathrm{a}$ & $9.8 \mathrm{a}$ & $6.9 \mathrm{a}$ & $8.5 \mathrm{a}$ & $6.4 \mathrm{a}$ & $6.6 \mathrm{a}$ & $6.9 \mathrm{a}$ & $7.7 \mathrm{a}$ & $8.3 a$ & $7.3 \mathrm{a}$ & $8.0 \mathrm{a}$ & $5.7 \mathrm{a}$ & $7.6 a$ & $5.2 \mathrm{a}$ & 4.7 \\
\hline \multicolumn{17}{|c|}{ Trial II } \\
\hline 55 & 22.1ce & $20.5 \mathrm{ce}$ & $21.7 \mathrm{de}$ & $22.7 \mathrm{ac}$ & $21.8 \mathrm{ec}$ & $23.7 \mathrm{ac}$ & $24.7 \mathrm{a}$ & $24.6 \mathrm{a}$ & $22.3 \mathrm{ec}$ & $24.1 \mathrm{ab}$ & $24.2 \mathrm{ab}$ & $23.6 \mathrm{ac}$ & $21.1 \mathrm{f}$ & $21.5 \mathrm{ed}$ & $14.4 \mathrm{~g}$ & 1.9 \\
\hline 62 & $20.4 \mathrm{ab}$ & $20.4 \mathrm{ab}$ & $21.2 \mathrm{ab}$ & $21.5 \mathrm{a}$ & $17.5 b$ & $21.8 \mathrm{a}$ & $20.3 \mathrm{ab}$ & $21.8 \mathrm{a}$ & $23.3 \mathrm{ac}$ & $22.4 \mathrm{~cd}$ & $23.1 \mathrm{ac}$ & $21.5 \mathrm{ce}$ & $19.8 \mathrm{f}$ & $21.5 \mathrm{ec}$ & $14.6 \mathrm{~g}$ & 4.3 \\
\hline 69 & $13.5 \mathrm{ab}$ & $14.3 \mathrm{ab}$ & $13.6 \mathrm{ab}$ & $21.5 \mathrm{ab}$ & $13.6 \mathrm{ab}$ & $11.2 \mathrm{bc}$ & $13.5 \mathrm{ab}$ & $15.5 \mathrm{ab}$ & $12.8 \mathrm{ac}$ & $16.5 \mathrm{a}$ & $13.1 \mathrm{bc}$ & $18.3 \mathrm{ab}$ & $15.5 \mathrm{ab}$ & $13.3 \mathrm{ab}$ & $6.9 c^{\circ}$ & 5.3 \\
\hline 76 & $11.8 \mathrm{~cd}$ & $15.7 \mathrm{ac}$ & $19.5 \mathrm{a}$ & $13.4 \mathrm{bc}$ & $15.8 \mathrm{ac}$ & $18.5 \mathrm{ab}$ & $17.9 \mathrm{ab}$ & $16.7 \mathrm{ac}$ & $16.3 \mathrm{ac}$ & $19.3 \mathrm{a}$ & $13.6 \mathrm{bc}$ & $18.3 \mathrm{ab}$ & $15.6 \mathrm{ac}$ & $15.2 \mathrm{ac}$ & $6.3 \mathrm{~d}$ & 4.4 \\
\hline 83 & $7.3 \mathrm{a}$ & $8.2 \mathrm{a}$ & $9.4 \mathrm{a}$ & $6.3 \mathrm{a}$ & $8.2 \mathrm{a}$ & $6.2 \mathrm{a}$ & $6.2 \mathrm{a}$ & $6.2 \mathrm{a}$ & $7.2 \mathrm{a}$ & $8.1 \mathrm{a}$ & 7.1a & $7.8 \mathrm{a}$ & $5.9 \mathrm{a}$ & $7.5 \mathrm{a}$ & $5.3 \mathrm{a}$ & 4.5 \\
\hline
\end{tabular}

* Means with the same letter(s) along the row for DAP and the column for Extracts are not significantly different at $\mathrm{P} \leq 0.05$ by Tukey's test. KEY: $\mathrm{N}=$ Neem, $\mathrm{T}=$ Tithonia, Te $=$ Tephrosia, Oxy =Oxamyl, $\mathrm{CNO}=\mathrm{Control}, \mathrm{C}$ with $=$ Control with nematodes, the numbers 25,50 , and 100 represent concentration of extract in $\mathrm{ml} / \mathrm{L}$

Table 3. Effects of Different Extracts on Lath house Pods Weight

\begin{tabular}{|c|c|c|c|c|c|c|c|c|c|c|c|c|c|c|c|c|}
\hline \multicolumn{17}{|c|}{ Extracts Concentration Rate } \\
\hline DAE & $\mathrm{N} 25$ & N50 & N100 & $\mathrm{Nb} 25$ & $\mathrm{Nb50}$ & $\mathrm{Nb} 100$ & $\mathrm{~T} 25$ & T50 & $\mathrm{T} 100$ & $\mathrm{Te} 25$ & $\mathrm{Te} 50$ & Te100 & Oxy & $\mathrm{CNO}$ & CWith & MSD \\
\hline \multicolumn{17}{|c|}{ Trial I } \\
\hline 55 & $59.3 \mathrm{a}^{*}$ & 64.1a & $61.6 a$ & $55.6 \mathrm{ab}$ & $59.5 \mathrm{a}$ & $61.5 \mathrm{a}$ & $63.4 \mathrm{a}$ & $58.5 \mathrm{a}$ & $44.8 \mathrm{ab}$ & $56.6 \mathrm{ab}$ & $56.4 \mathrm{ab}$ & $62.1 \mathrm{a}$ & $62.2 \mathrm{a}$ & $57.8 \mathrm{a}$ & $35.5 b$ & 21.2 \\
\hline 62 & $64.1 \mathrm{a}$ & $63.7 \mathrm{a}$ & $64.1 \mathrm{a}$ & $63.5 \mathrm{a}$ & $57.4 \mathrm{a}$ & $65.6 \mathrm{a}$ & $54.1 \mathrm{a}$ & $58.2 \mathrm{a}$ & $58.9 \mathrm{a}$ & $59.7 \mathrm{a}$ & $57.6 \mathrm{a}$ & $55.8 \mathrm{a}$ & $57.6 \mathrm{a}$ & $58.2 \mathrm{a}$ & $30.5 b$ & 27.05 \\
\hline 69 & $50.7 \mathrm{ab}$ & $52.8 \mathrm{ab}$ & $45.7 \mathrm{ab}$ & $56.4 \mathrm{a}$ & $53.6 \mathrm{ab}$ & $46.8 \mathrm{ab}$ & $51.4 \mathrm{ab}$ & $55.5 \mathrm{ab}$ & $59.8 \mathrm{a}$ & $54.4 \mathrm{ab}$ & $49.7 \mathrm{ab}$ & $28.6 \mathrm{bc}$ & $43.5 \mathrm{ab}$ & $46.3 \mathrm{ab}$ & $15.5 \mathrm{c}$ & 27.05 \\
\hline 76 & $46.5 \mathrm{a}$ & $48.2 \mathrm{a}$ & $53.7 \mathrm{a}$ & $22.9 b$ & $50.6 \mathrm{a}$ & $46.4 \mathrm{ab}$ & $46.2 \mathrm{ab}$ & $44.9 \mathrm{ab}$ & $48.4 \mathrm{a}$ & $49.8 \mathrm{a}$ & $48.4 \mathrm{a}$ & $52.6 \mathrm{a}$ & $45.3 \mathrm{ab}$ & $53.3 \mathrm{a}$ & $30.1 \mathrm{ab}$ & 26.0 \\
\hline 83 & $31.2 \mathrm{a}$ & $25.9 \mathrm{a}$ & $27.7 \mathrm{a}$ & $22.9 \mathrm{ab}$ & $29.0 \mathrm{a}$ & $23.5 \mathrm{a}$ & $15.8 \mathrm{ab}$ & $17.7 \mathrm{ab}$ & $21.4 \mathrm{ab}$ & $26.0 \mathrm{a}$ & $26.3 \mathrm{a}$ & $54.4 \mathrm{ab}$ & $19.3 \mathrm{ab}$ & $4.16 b$ & $28.6 \mathrm{bc}$ & 30.5 \\
\hline \multicolumn{17}{|c|}{ Trial II } \\
\hline 55 & $55.3 \mathrm{a}$ & $64.1 \mathrm{a}$ & $61.6 \mathrm{a}$ & $55.6 \mathrm{ab}$ & $59.5 a$ & $61.5 \mathrm{a}$ & $63.4 \mathrm{a}$ & $58.5 \mathrm{a}$ & $44.8 \mathrm{ab}$ & $56.6 \mathrm{ab}$ & $56.4 \mathrm{ab}$ & $62.1 \mathrm{a}$ & $62.2 \mathrm{a}$ & $57.8 \mathrm{a}$ & $35.5 b$ & 21.2 \\
\hline 62 & $64.1 \mathrm{a}$ & $63.7 \mathrm{a}$ & $64.1 \mathrm{a}$ & $63.5 \mathrm{a}$ & $57.4 \mathrm{a}$ & $65.6 \mathrm{a}$ & $54.1 \mathrm{a}$ & $58.2 \mathrm{a}$ & $58.9 \mathrm{a}$ & $59.7 \mathrm{a}$ & $57.6 \mathrm{a}$ & $55.8 \mathrm{a}$ & $57.6 \mathrm{a}$ & $58.2 \mathrm{a}$ & $30.5 b$ & 27.05 \\
\hline 69 & $51.7 \mathrm{ab}$ & $52.8 \mathrm{ab}$ & $45.7 \mathrm{ab}$ & $56.4 \mathrm{a}$ & $53.6 \mathrm{ab}$ & $46.8 \mathrm{ab}$ & $51.4 \mathrm{ab}$ & $55.5 \mathrm{ab}$ & $59.8 \mathrm{a}$ & $54.4 \mathrm{ab}$ & $49.7 \mathrm{ab}$ & $28.6 b c$ & $43.5 \mathrm{ab}$ & $46.3 \mathrm{ab}$ & $15.5 \mathrm{c}$ & 27.05 \\
\hline 76 & $46.5 \mathrm{a}$ & $48.2 \mathrm{a}$ & $53.7 \mathrm{a}$ & $22.9 b$ & $50.6 \mathrm{a}$ & $46.4 \mathrm{ab}$ & $46.2 \mathrm{ab}$ & $44.9 \mathrm{ab}$ & $48.4 \mathrm{a}$ & $49.8 \mathrm{a}$ & $48.4 \mathrm{a}$ & $52.6 \mathrm{a}$ & $45.3 \mathrm{ab}$ & $53.3 \mathrm{a}$ & $30.1 \mathrm{ab}$ & 26.0 \\
\hline 83 & $31.2 \mathrm{a}$ & $25.9 \mathrm{a}$ & $27.7 \mathrm{a}$ & $22.9 \mathrm{ab}$ & $29.0 \mathrm{a}$ & $23.5 \mathrm{a}$ & $15.8 \mathrm{ab}$ & $17.7 \mathrm{ab}$ & $21.4 \mathrm{ab}$ & $26.0 \mathrm{a}$ & $26.3 \mathrm{a}$ & $54.4 \mathrm{ab}$ & $19.3 \mathrm{ab}$ & $4.16 \mathrm{~b}$ & $28.6 \mathrm{bc}$ & 30.5 \\
\hline
\end{tabular}

*Means with the same letter(s) along the row for DAP and the column for Extracts are not significantly different at $\mathrm{P} \leq 0.05$ by Tukey's test. KEY: $\mathrm{N}=$ Neem, $\mathrm{T}=$ Tithonia, $\mathrm{Te}=$ Tephrosia, $\mathrm{Oxy}=\mathrm{Oxamyl}, \mathrm{CNO}=\mathrm{Control}, \mathrm{C}$ with $=$ Control with nematodes, the numbers 25,50 , and 100 represent concentration of extract in $\mathrm{ml} / \mathrm{L}$

\section{Root Galling in a Field Experiment}

The results of these study indicated that root-knot nematodes galling indices among the extracts showed were significantly different $(P=0.05)$ with the untreated control recording the highest root galling index at 10.3 and 10.6 galls in trial I and trial II, respectively. The highest reduction in root galling index was recorded under the plots treated with Neem at both 50 $\mathrm{ml} / \mathrm{L}$ and $100 \mathrm{ml} / \mathrm{L}$ at 1 gall in both trial I and trial II. 3.3 and 3.1 galls were observed in Oxymyl (positive synthetic control) treated plots in trial I and trial II, respectively (Table 5).

\section{Number of Pods in Lath house Potted Plants}

It was observed that French beans number of pods among different crude extract treatments 
differed significantly $(P=0.05)$ at 55,63, 69 and 76 DAE .The results of this study showed that crude extracts treated French beans plants produced significantly higher French bean number of pods compared to untreated controls infested with root-knot nematodes. From the lath house study the untreated control attained the least number of pods at 9.5 and 9.7 pods in trial I and trial II, respectively (Table 5).

Table 4. Effects of Different Extracts on Field Pods Weight

\begin{tabular}{|c|c|c|c|c|c|c|c|c|c|c|c|c|c|c|c|c|}
\hline \multicolumn{17}{|c|}{ Extracts Concentration Rate } \\
\hline DAE & $\mathrm{N} 25$ & $\mathrm{~N} 50$ & N100 & $\mathrm{Nb} 25$ & $\mathrm{Nb50}$ & $\mathrm{Nb} 100$ & $\mathrm{~T} 25$ & T50 & T100 & $\mathrm{Te} 25$ & Te50 & $\mathrm{Te} 100$ & Oxy & $\mathrm{CNO}$ & CWith & MSD \\
\hline \multicolumn{17}{|c|}{ Trial I } \\
\hline 55 & $67.0 \mathrm{a}^{*}$ & $73.4 \mathrm{a}$ & $71.1 \mathrm{a}$ & $72.0 \mathrm{a}$ & $70.2 \mathrm{a}$ & $74.1 \mathrm{a}$ & $73.2 \mathrm{a}$ & $70.9 \mathrm{a}$ & $71.1 \mathrm{a}$ & $67.7 \mathrm{a}$ & $69.2 \mathrm{a}$ & $74.0 \mathrm{a}$ & $69.2 \mathrm{a}$ & $69.2 \mathrm{a}$ & $29.9 b$ & 18.5 \\
\hline 62 & $69.2 \mathrm{a}$ & $71.0 \mathrm{a}$ & $73.3 \mathrm{a}$ & $73.4 \mathrm{a}$ & $62.6 \mathrm{a}$ & $72.6 a$ & $67.4 \mathrm{a}$ & $66.8 \mathrm{a}$ & $73.0 \mathrm{a}$ & $72.5 \mathrm{a}$ & $69.0 \mathrm{a}$ & $65.2 \mathrm{a}$ & $69.2 \mathrm{a}$ & $68.9 \mathrm{a}$ & $32.4 \mathrm{~b}$ & 19.0 \\
\hline 69 & $54.3 \mathrm{a}$ & $48.1 \mathrm{a}$ & $47.3 \mathrm{a}$ & $49.7 \mathrm{a}$ & $50.0 \mathrm{a}$ & $46.2 \mathrm{a}$ & $46.9 \mathrm{a}$ & $51.9 \mathrm{a}$ & $44.3 \mathrm{a}$ & $52.9 \mathrm{a}$ & $51.5 \mathrm{a}$ & $53.1 \mathrm{a}$ & $48.2 \mathrm{a}$ & $53.82 \mathrm{a}$ & $26.7 b$ & 16.4 \\
\hline 76 & $57.1 \mathrm{a}$ & $63.3 \mathrm{a}$ & $65.5 \mathrm{a}$ & $63.7 \mathrm{a}$ & $64.2 \mathrm{a}$ & $66.5 a$ & $63.2 \mathrm{a}$ & $63.3 \mathrm{a}$ & $58.09 \mathrm{a}$ & $62.0 \mathrm{a}$ & $60.1 \mathrm{a}$ & $64.2 \mathrm{a}$ & $64.3 \mathrm{a}$ & $57.7 \mathrm{a}$ & $20.2 b$ & 16.7 \\
\hline 83 & $35.2 \mathrm{ab}$ & $37.5 \mathrm{ab}$ & $42.1 \mathrm{ab}$ & $37.5 \mathrm{ab}$ & $41.4 \mathrm{ab}$ & $43.1 \mathrm{a}$ & $38.0 \mathrm{ab}$ & $40.4 \mathrm{ab}$ & $39.1 \mathrm{ab}$ & $41.3 \mathrm{ab}$ & $39.4 \mathrm{ab}$ & $42.0 \mathrm{ab}$ & $40.5 \mathrm{ab}$ & $39.2 \mathrm{ab}$ & $32.3 b$ & 20.1 \\
\hline \multicolumn{17}{|c|}{ Trial II } \\
\hline 55 & $67.1 \mathrm{a}$ & $73.2 \mathrm{a}$ & $71.2 \mathrm{a}$ & $72.2 \mathrm{a}$ & $70.1 \mathrm{a}$ & $73.3 \mathrm{a}$ & $70.7 \mathrm{a}$ & $71.3 \mathrm{a}$ & $72.2 \mathrm{a}$ & $72.2 \mathrm{a}$ & $72.1 \mathrm{a}$ & $75.8 \mathrm{a}$ & $72.1 \mathrm{a}$ & $70.3 \mathrm{a}$ & $30.5 b$ & 19.6 \\
\hline 62 & $69.5 a$ & $71.4 \mathrm{a}$ & $73.5 \mathrm{a}$ & $73.5 \mathrm{a}$ & $63.4 \mathrm{a}$ & 73.5 & $68.9 \mathrm{a}$ & $65.9 \mathrm{a}$ & $73.4 \mathrm{a}$ & $74.5 \mathrm{a}$ & $69.7 \mathrm{a}$ & $66.8 \mathrm{a}$ & $70.1 \mathrm{a}$ & $69.5 \mathrm{a}$ & $33.2 b$ & 18.6 \\
\hline 69 & $53.3 \mathrm{a}$ & $47.5 \mathrm{a}$ & $46.3 \mathrm{a}$ & $48.6 \mathrm{a}$ & $49.3 a$ & $45.8 \mathrm{a}$ & $50.1 \mathrm{a}$ & $48.7 \mathrm{a}$ & $44.6 \mathrm{a}$ & $51.2 \mathrm{a}$ & $50 \mathrm{a}$ & $62.6 \mathrm{a}$ & $48.2 \mathrm{a}$ & $53.82 \mathrm{a}$ & $46.9 \mathrm{~b}$ & 16.8 \\
\hline 76 & $57.1 \mathrm{a}$ & $63.3 \mathrm{a}$ & $65.5 \mathrm{a}$ & $63.7 \mathrm{a}$ & $64.2 \mathrm{a}$ & $66.5 a$ & $63.2 \mathrm{a}$ & $63.3 \mathrm{a}$ & $58.09 \mathrm{a}$ & $62.0 \mathrm{a}$ & $60.1 \mathrm{a}$ & $64.2 \mathrm{a}$ & $64.3 \mathrm{a}$ & $57.7 \mathrm{a}$ & $20.2 b$ & 35.2 \\
\hline 83 & $35.2 \mathrm{ab}$ & $37.5 \mathrm{ab}$ & $42.1 \mathrm{ab}$ & $37.5 \mathrm{ab}$ & $41.4 \mathrm{ab}$ & $43.1 \mathrm{a}$ & $38.0 \mathrm{ab}$ & $40.4 \mathrm{ab}$ & $39.1 \mathrm{ab}$ & $41.3 \mathrm{ab}$ & $39.4 \mathrm{ab}$ & $42.0 \mathrm{ab}$ & $40.5 \mathrm{ab}$ & $39.2 \mathrm{ab}$ & $32.3 \mathrm{~b}$ & 19.0 \\
\hline
\end{tabular}

*Means with the same letter(s) along the row for DAP and the column for Extracts are not significantly different at $\mathrm{P} \leq 0.05$ by Tukey's test. KEY: $\mathrm{N}=$ Neem, $\mathrm{T}=$ Tithonia, Te $=$ Tephrosia, $\mathrm{Oxy}=\mathrm{Oxamyl}, \mathrm{CNO}=\mathrm{Control}, \mathrm{C}$ with $=$ Control with nematodes, the numbers 25,50 , and 100 represent concentration of extract in $\mathrm{ml} / \mathrm{L}$

Table 5. Effects of Different Extracts on Root galling Indices in the lath house and field experiment

\begin{tabular}{|c|c|c|c|c|c|c|c|c|c|c|c|c|c|c|c|}
\hline \multicolumn{16}{|c|}{ Lath house } \\
\hline \multicolumn{16}{|c|}{ Extracts Concentration Rate } \\
\hline N25 & N50 & N100 & $\mathrm{Nb} 25$ & Nb50 & $\mathrm{Nb} 100$ & $\mathrm{~T} 25$ & T50 & T100 & Te25 & Te50 & Te100 & Oxy & $\mathrm{CNO}$ & CWith & MSD \\
\hline \multicolumn{16}{|l|}{ Trial I } \\
\hline$* 1 \mathrm{c}$ & $1.6 \mathrm{c}$ & $1 \mathrm{c}$ & $1.8 \mathrm{c}$ & $1.7 \mathrm{c}$ & $2.7 \mathrm{c}$ & $2.4 \mathrm{c}$ & $2.8 \mathrm{c}$ & $2.8 \mathrm{c}$ & $4.6 \mathrm{bc}$ & $3.4 \mathrm{bc}$ & $1.9 \mathrm{c}$ & $1.8 \mathrm{c}$ & 1.1.c & $14.4 \mathrm{a}$ & 3.2 \\
\hline \multicolumn{16}{|l|}{ Trial II } \\
\hline$* 1.5 \mathrm{c}$ & $1.6 \mathrm{c}$ & $1.5 \mathrm{c}$ & $1.9 \mathrm{c}$ & $1.9 \mathrm{c}$ & $2.8 \mathrm{c}$ & $2.4 \mathrm{c}$ & $2.8 \mathrm{c}$ & $2.8 \mathrm{c}$ & $4.9 \mathrm{bc}$ & $3.4 \mathrm{bc}$ & $2.1 \mathrm{c}$ & $1.8 \mathrm{c}$ & 1.4.c & $17.4 \mathrm{a}$ & 2.9 \\
\hline \multicolumn{16}{|c|}{ Field Experiment } \\
\hline \multicolumn{16}{|c|}{ Extracts Concentration Rate } \\
\hline N25 & N50 & N100 & $\mathrm{Nb} 25$ & $\mathrm{Nb50}$ & $\mathrm{Nb} 100$ & $\mathrm{~T} 25$ & T50 & $\mathrm{T} 100$ & Te25 & Te50 & Te10 & Oxy & $\mathrm{CNO}$ & CWith & MSD \\
\hline \multicolumn{16}{|l|}{ Trial I } \\
\hline $\begin{array}{l}2.4 \mathrm{~b} c^{*} \\
\text { Trial II }\end{array}$ & $2.1 \mathrm{bc}$ & $2.1 \mathrm{bc}$ & $1.1 \mathrm{c}$ & $1.0 \mathrm{c}$ & $1.5 \mathrm{c}$ & $1.1 \mathrm{c}$ & $3.13 b c$ & $4.8 \mathrm{~b}$ & $3.0 \mathrm{bc}$ & $3.2 \mathrm{bc}$ & $2.7 \mathrm{bc}$ & $3.3 \mathrm{bc}$ & $3.4 \mathrm{cb}$ & $10.3 \mathrm{a}$ & 2.9 \\
\hline $2.1 \mathrm{bc}$ & $1.2 \mathrm{~b}$ & $1.2 \mathrm{~b}$ & $1.4 \mathrm{bc}$ & $1.2 \mathrm{c}$ & $1.4 \mathrm{c}$ & $1.7 \mathrm{c}$ & $2.4 \mathrm{bc}$ & $2.3 \mathrm{~b}$ & $2.5 \mathrm{bc}$ & $2.4 \mathrm{~b}$ & $2.2 \mathrm{bc}$ & $3.0 \mathrm{~b}$ & $3.0 \mathrm{~b}$ & $10.8 \mathrm{a}$ & 2.6 \\
\hline
\end{tabular}

*Means with the same letter(s) along the row for DAP and the column for Extracts are not significantly different at $\mathrm{P} \leq 0.05$ by Tukey's test. KEY: $\mathrm{N}=$ Neem, $\mathrm{T}=$ Tithonia, Te $=$ Tephrosia, $\mathrm{Oxy}=\mathrm{Oxamyl}, \mathrm{CNO}=\mathrm{Control}, \mathrm{C}$ with $=$ Control with nematodes, the numbers 25,50 , and 100 represent concentration of extract in $\mathrm{ml} / \mathrm{L}$

\section{Discussion}

\section{Effects of Different Extracts on French bean Yield}

The presence of the nematodes on the French bean plants significantly affected their yield. Crude plant extract inoculated plants had 50\% higher yields than plants which were not inoculated with the extracts. Yield in terms of pod number and pod weight from French beans treated with the crude plant extracts were significantly better than yields from plants treated 
with Oxamyl. The findings of this study also agree with those of Sidhu et al. (2017) who reported that application of botanical extracts increases yields and may help manage root-knot nematode populations in infested fields. Neem, Tithonia and Tephrosia extracts above their ability to suppress plant parasitic nematodes by changing the soil chemical properties, probably also changed the physical properties and enriched the soil with beneficial microflora.

These changes in the soil properties probably negatively affected the growth or multiplication of the nematodes in the French beans. The findings of this research are also in agreement with those of Aiyadurai et al. (2018) who suggested that plant parasitic nematodes can be controlled by application of botanicals. Water and nutrition are important vital components in French bean production life and their limited supply or unavailability is one of the major abiotic factors that adversely affect French beans production in many ways. Proper nutrition is the basic need of every living organism as the nutrients are not only required for better plant growth and development, but they are also helpful to alleviate different kinds of abiotic stresses like drought stress. However, recent trends indicate that productivity and fertility of soils are globally declining due to degradation and intensive use of soils without consideration of proper soil management practices and use of synthetic chemicals (Muhammad et al., 2017).

Studies by Sikora et al. (2018) indicated that root-knot nematodes inhibits or slows down photosynthetic carbon fixation which directly inhibit plant metabolism. It is possible that French bean plants treated with higher crude extract levels compared to low extract levels and untreated plants experienced better and high metabolism and consequently better growth. From the study it was noted that the crude extracts significantly increased the pod number and pod weight of French bean plants per plant when compared to the untreated control. The results of this study agree with those by Rajesh et al. (2017) who reported that crude extracts from Neem leaf extracts at $100 \mathrm{ml} / \mathrm{L}$ and Tithonia $100 \mathrm{ml} / \mathrm{L}$ were most effective and increased the yield significantly.

From the study it was noted that the increase in French bean yield was more when compared with the chemical nematicides. Neembecidine leaf extracts at all levels of concentration followed the Neem in improving the French beans yield. The least yield was obtained from the severely infested untreated control. In all botanical extracts application, yields of French bean per plant increased with the increase in concentration. On average Tephrosia leaf extracts, were the least effective in increasing the yield of French bean per plant.

The importance of organic horticultural production, which avoids synthetic nematicides applications, increased the research on botanical pesticides with potential use for nematode management (Mishra et al., 2018). In this study application of plant extract was found to reduce root-knot nematode galling indices on French beans root system and thus the final nematode population density in the soil was significantly lowered over the untreated control. With the increase in level of botanical extract concentration, a corresponding significant reduction was observed in the number of galls and nematode population over untreated control. Root-knot infestation stunted all untreated plants and reduced leaf production as well as French bean yield. 
The effects of the tested botanical extracts against infestation of root-knot nematode and yield of French bean plants were different, in some cases. The differences in the toxicity of different botanical extracts could be due to the differences in the chemical compositions and concentrations of toxic components. Crude extract treated plots when compared to the positive control (Oxamyl) a synthetic nematicide, controls yielded significantly heavier pod weight at harvest. Also extract treatments gave a $100 \%$ increase in both pod number and pod weight over the untreated control in which most of the plants withered away and died. These yield benefits can be attributed to increased root knot nematode control in the soil by the crude extracts amendments.

Organic amendments have been reported to improve plant tolerance to nematode damage and in turn promote better yields (Pavaraj et al., 2018). Although Oxamyl suppressed root-knot nematodes RKN population satisfactorily, lower results were obtained when compared to the crude extracts treatment application observed in terms of crop yield. The lower results in Oxamyl, when compared to crude extracts, could be attributed to the fact that Oxamyl is a broad-spectrum fumigant in terms of activity and thus could have eliminated all the beneficial soil micro-organisms that would have been useful in checking the nematode populations and also in maintaining the soil biology.

\section{Effects of Different Extracts on Root galling Indices}

The extent of galling on roots (root-knot index) is a means for detecting the infestation of Meloidogyne species in the roots and the damage or severity caused. In this study, all the Plant extracts were highly effective in reducing root-knot index when compared with untreated plants. The extracts reduced root galling significantly and were at par with Oxamyl (synthetic nematicides). The study showed that among the crude extracts treatment evaluated, there were variations in root-knot nematode damages.

The low galling indices in extract treated control treatments indicated low damages on the roots in French beans. Root gall index appeared to be a useful parameter in evaluating the efficacy of the crude extracts formulation on Meloidogyne indica. The study revealed that the extracts evaluated improved plant status, exerted significant control on the root-knot nematodes and reduced the root galling indices in French beans. It was observed that French bean performance was best in plants treated with Neem and closely followed by those treated with neembecidine. These results agree with those obtained results and are in agreement with findings by Pavaraj et al. (2018) who reported that leaf extracts of Neem, Tithonia and Tephrosia significantly reduce root-knot nematodes.

\section{Conclusion}

The study provides empirical data confirming the use of botanicals for improved French bean production as a food security crop, although the treatments are in high demand in ethno medicinal practices, they appear to have a higher potential in the control of $M$. incognita and have proved to be favorable alternatives to synthetic nematicides pesticides in vegetable crop production. The introduction of crude botanical extracts as an option in the management of parasitic nematodes has become a major component in sustainable management of soil health 
and productivity. Eco-friendly nematicides used in the experiment led to root-knot nematode suppression which did not differ significantly from Vydate (synthetic nematicides). This study illustrates that agricultural utilization of phytochemicals although currently under trial and development in many situations, offers tremendous potential in the control of root-knot nematodes. Although the plants in this study are locally used in ethno medicinal practices without toxicological residue effects and as such could serve as alternatives in a sustainable organic farming system to meet global demand in food production.

\section{Recommendations}

Since botanical extracts are effective in root-knot nematode suppression, they are recommended for incorporation into integrated nematode management in French bean production. Since the extracts were comparable to synthetic nematicides in root-knot nematode management, they can be considered for use as an alternative. More studies on the integration of botanical extracts and other control agents on the activities of plant parasitic nematodes and other soil borne pests in crop production systems are highly recommended.

\section{Acknowledgements}

The authors appreciate the USAID support through KALRO and Chuka University Internal Research grant for financing the field work.

\section{References}

Aiyadurai, N., Muthu, M., Vaganan, S., \& Rajangam, U. (2018). Nematicidal Activity of Aqueous Leaf Extracts of Datura metel, Datura innoxia and Brugmansia suaveolens, American Journal of Entomology, 1(2), 39-45.

https://doi.org/10.9734/AJOB/2017/34241

Coyne, D. L., Cortada, L., Dalzell, J. J., Claudius-Cole, A. O., Haukeland, S., Luambano, N., \& Talwana, H. (2018). Plant-Parasitic Nematodes and Food Security in Sub-Saharan Africa. Annual Review of Phytopathology. 56, 381-403.

https://doi.org/10.1146/annurev-phyto-080417-045833

Dougoud, J., Toepfer, S., Bateman, M., \& Jenne, W. H. (2019). Efficacy of homemade botanical insecticides based on traditional knowledge. A review. Agronomy for Sustainable Development, 39, 37. https://doi.org/10.1007/s13593-019-0583-1

Emmanuel, O., Solveig, H., \& Wim, B. (2017). Efficacy of Commercial and Non-Commercial Fungal Isolates for Suppression of Root-Knot Nematode on Pineapple. International Centre of Insect Physiology and Ecology.

Jang, J. Y., Dang, Q., Choi, L., Park, G. J., \& Kim, J. C. (2019). Control of root-knot nematodes using Waltheria indica producing 4-quinolone alkaloids. Pest Management Science, 75, 2264-2270. https://doi.org/10.1002/ps.5363

Mishra, S. D. (2018). Effects of an aqueous extracts of green leaves of neem, madar and tulsi on the hatching of eggs of root-knot nematode Meloidogyne incognita. Current Nematology, 7(2), 133-135. 
Muhammad, A., Azeem, F., Abbas, A., Joyia, F. A., Li, H., \& Dababat, A. A. (2017). Transgenic strategies for enhancement of nematode resistance in plants. Frontiers in Plant Science, 8, 750. https://doi.org/10.3389/fpls.2017.00750

Odeyemi, S., \& Adewale, A. (2011). Phythonematoxic properties and nematicidal potential of Tithonia diversifolia extract and residue on Meloidogyne incognita infecting yam (Discoria rotundata). Archives of Phytopathology and Plant

Protection, 44(18), 1745-1753. https://doi.org/10.1080/03235400903461039

Oloruntola, D. A., Dada, E. O., \& Osho, I. B. (2017). Effects of Hydro-ethanolic Leaf Extract of Tithonia diversifolia on Parasitaemia Level. Asian Journal of Medicine and Health, 6(2), 1-11. https://doi.org/10.9734/AJMAH/2017/34048

Oyinlola, A. A. (2017). Allelopathic Potentials Of Aqueous Extracts of Tithonia Diversifolia (Hemsley) A. Gray in biological control of weeds in cowpea cropping system. International Journal of Agriculture and Economic Development, 5(1), 11-28.

Patil, S. Y., \& Sharma, M. K. (2017). Effect of Plant Extracts on Egg Hatching and Juvenile Mortality of Reniform Nematode, Rotylenchulus reniformis. International Journal of Pure and Applied Bioscience, 5(2), 158-164. https://doi.org/10.18782/2320-7051.2813

Pavaraj, M., Bakavathia, G. G., \& Baskaran, S. (2018). Evaluation of some plant extracts for their nematicidal properties against root-knot nematode, Meloidogyne incognita. Journal of Biopesticides (Supplementary), 106-110.

Perry, R. N., \& Evans, A. F. (2009). Survival mechanisms in Root-knot nematodes, 201-220. $\mathrm{CAB}$ international. London.

Priya, M. S., \& Pandiyan, M. (2019). Efficacy of Botanical Extracts on Hatching of Meloidogyne incognita Eggs under in vitro Study. International Journal of Current Microbiology and Applied Sciences, 8(1), 2664-2668.

https://doi.org/10.20546/ijcmas.2019.801.280

Rajesh, A., \& Alfred, D. (2017). Response of Neem Cake Rhizobium and Inorganic Fertilizer on Soil Health Growth and Yield of Green gram (Vigna radiate L.) var. Samrat. International Journal of Current Microbiology and Applied Sciences, 6(7), 815-821.

https://doi.org/10.20546/ijcmas.2017.607.100

Sidhu, H., Singh, V. K., \& Madhu, M. R. (2017). Eco-Friendly Management of Root-knot Nematode, Meloidogyne javanica in Okra (Abelmoschus esculentus) crop. International Journal of Pure and Applied Bioscience, 5(1), 569-574.

https://doi.org/10.18782/2320-7051.2507

Sikora, R. A., \& Fernandez, E. (2018). Nematode parasites of vegetables, In: Luc, M., Sikora, R.A, Bridge, J. (Eds.). Plant Parasitic Nematodes in Subtropical and Tropical Agriculture, P. 319-392. CABI Publishing, Wallingford, UK. https://doi.org/10.1079/9780851997278.0319

Sujatha, R., Irene Vethamoni, P., Manivannan, N., \& Sivakuma, M. (2017). Screening of Tomato genotypes for root-knot nematode (Meloidogyne incognita Kofoid and White 


\section{Macrothink}

Journal of Agricultural Studies

ISSN 2166-0379 2019, Vol. 7, No. 3

Chitwood). International Journal of Current Microbiology and Applied Sciences, 6(3), 1525-1533. https://doi.org/10.20546/ijcmas.2017.603.175

Wachira, P. M., Kimenju, J. W., \& Okoth, S. A. (2009).Stimulation of nematode-destroying fungi by organic amendments applied in management of plant parasitic nematodes. Asian Journal of Plant Science, 2, 153-159. https://doi.org/10.3923/ajps.2009.153.159

\section{Copyright Disclaimer}

Copyright for this article is retained by the author(s), with first publication rights granted to the journal.

This is an open-access article distributed under the terms and conditions of the Creative Commons Attribution license (http://creativecommons.org/licenses/by/4.0/). 